\title{
The Effect of Daily Intraperitoneal Injection of Deferoxamine in an Acute Model of Cisplatin Induced Nephrotoxicity
}

\author{
Mai M. Abdelmageed ${ }^{a}$, Eman M. Mantawy ${ }^{b}$, Reem N. El-Naga ${ }^{b}$, Samar S. Azab ${ }^{b}$, Ebtehal El-Demerdash ${ }^{b}$, \\ Mohamed M. Elmazar*a \\ ${ }^{a}$ Department of Pharmacology and Toxicology, Faculty of Pharmacy, The British University in Egypt (BUE), Cairo \\ 11837, Egypt \\ ${ }^{\mathrm{b}}$ Department of Pharmacology and Toxicology, Faculty of Pharmacy, Ain Shams University, Cairo 11566, Egypt
}

\begin{abstract}
Cisplatin is a major classical anticancer agent. However, cisplatin-induced nephrotoxicity remains a major doselimiting side effect. Several studies have highlighted the role of catalytic iron content in several diseases including cisplatin nephrotoxicity opening the doors for adding iron chelators as an adjunct therapy to cisplatin therapy. Since deferoxamine is the major approved chelation therapy in iron overload disorders, the current study was directed to exploring the possible nephroprotective outcomes of deferoxamine in an acute animal model of cisplatin-induced renal injury. Male Sprague-Dawley rats were injected i.p. daily for 6 consecutive days with 3 different dose levels of deferoxamine as follows: 100, 200, and $300 \mathrm{mg} / \mathrm{kg}$. On day 3, cisplatin was injected i.p. as a single dose of 7.5 $\mathrm{mg} / \mathrm{kg}$, and animals were sacrificed on day 7. Measurement of serum creatinine and blood urea nitrogen (BUN) showed that iron chelation by deferoxamine failed to improve kidney function as demonstrated by the consistent high creatinine and BUN levels in all the treated groups. Calculation of kidney indices further confirmed the previous measurements. Histopathological examination of the renal tissues of animals showed the development of tubular degeneration in all treated groups indicating the absence of significant nephroprotection. In conclusion, our results show that the daily i.p. injection of deferoxamine is not protective against cisplatin-induced acute renal injury. However, the well-established body of evidence supporting the potential contribution of catalytic iron in cisplatin-induced nephrotoxicity establishes the demand for investigating the potential nephroprotective effects of other established iron chelation therapies.
\end{abstract}

Keywords: cisplatin; deferoxamine; acute renal injury; catalytic iron; intraperitoneal; chelation therapy.

*Correspondence | Mohamed M. Elmazar; Department of Pharmacology and Toxicology, Faculty of Pharmacy, The British University in Egypt (BUE), Cairo 11837, Egypt. Email: mohey.elmazar@bue.edu.eg

Citation | Abdelmageed MM, Mantawy EM, El-Naga RN, Azab SS, El-Demerdash E, Elmazar MM, 2020. The Effect of Daily Intraperitoneal Injection of Deferoxamine in an Acute Model of Cisplatin Induced Nephrotoxicity. Arch Pharm Sci ASU 4(2): 215-223

DOI: 10.21608 /aps.2020.42722.1040

Print ISSN: 2356-8380. Online ISSN: 2356-8399.

Received 22 September 2020. Accepted 15 October 2020.

Copyright: ${ }^{\circledR} 2020$ Abdelmageed et al. This is an open-access article licensed under a Creative Commons Attribution 4.0 International License (CC BY 4.0), which permits unrestricted use, distribution, and reproduction in any medium, provided the original author(s) and source are credited.

Published by: Ain Shams University, Faculty of Pharmacy

\section{INTRODUCTION}

Cisplatin (cis-diamminedichloroplatinum) is considered to be the primary heavy metal antineoplastic agent that is extensively added to the therapeutic regimen of numerous solid-organ malignant cancers, including lung, head, and neck, testicular and ovarian cancers [1]. Although cisplatin is a mainstay in the therapy of various cancers, many side effects were reported including nephrotoxicity, ototoxicity, and neurotoxicity; whereby nephrotoxicity is major dose-limiting toxicity occurring in approximately 
one-third of the patients $[2,3]$.

Iron is an essential macromineral that is involved in several vital biochemical processes including oxygen transport, the proliferation of cells, oxidative metabolism, and catalytic reactions [4]. Disturbance in iron metabolism has been linked to an increase in the catalytic reactive iron pool which subsequently contributes to the pathophysiology of several diseases including iron disorders [5] neurodegenerative diseases [6] lung diseases [7] and renal diseases [8]. Indeed, it has been shown that when the renal tubular epithelial cells are subjected to cisplatin, this, in turn, raises the catalytic iron pool which contributes to renal injury by increasing oxidative damage during cisplatin treatment and that the administration of iron chelators significantly attenuates cisplatin-induced nephrotoxicity [9].

Deferoxamine is the only injectable iron chelator available in the Egyptian market and is considered first-line therapy in iron overload disorders. Hence, the present study investigates whether a single daily intraperitoneal injection of the iron chelator "deferoxamine" could confer nephroprotection in an acute rat model of cisplatin-induced acute renal injury to address the feasibility of using this specific chelator in further mechanistic studies or potential clinical trials.

\section{MATERIALS AND METHODS}

\subsection{Animals}

Male Sprague-Dawley rats weighing between $140 \& 180 \mathrm{~g}$ were purchased from the animal unit of El-Nile Co., Cairo, Egypt. The rats were accommodated in standard animal cages that were kept under an air-conditioned atmosphere. The temperature was maintained at $25{ }^{\circ} \mathrm{C}$ and relative humidity was kept at $40-60 \%$ with alternative light and dark cycles changed at $12 \mathrm{~h}$ intervals at the animal research house of the School of Pharmacy, Ain Shams University,
Cairo, Egypt. Rats were allowed access to water and standard diet pellets freely. The standard animal diet was purchased from El-Nasr Chemical Co., Cairo, Egypt. The standard animal diet contained at least $20 \%$ protein, $6.5 \%$ ash, $5 \%$ fiber, $3.5 \%$ fat $\&$ a mixture of vitamins.

\subsection{Materials}

Cisplatin was obtained from the commercial vials available in the Egyptian market (Mylan, Cairo, Egypt). Deferoxamine was also purchased from the commercially available vials in Egypt (Novartis, Cairo, Egypt). All solvents and chemicals were obtained commercially of the highest available purity.

\subsection{Experimental design}

Rats were randomly assigned to five groups; each group had 10 animals. The animals received treatments for six days as follows; group 1 was injected the vehicle (saline) i.p. daily (negative control), group 2 was injected the vehicle (saline) i.p. daily except for the third day; where cisplatin was injected i.p. as a single dose of $7.5 \mathrm{mg} / \mathrm{Kg}$ to cause renal injury and groups 3,4 and 5 were injected deferoxamine i.p. daily for 6 consecutive days at doses of 100, 200 and $300 \mathrm{mg} / \mathrm{kg}$ for dose screening purposes and on the third-day cisplatin was injected i.p. as a single dose of $7.5 \mathrm{mg} / \mathrm{Kg}$ approximately $30 \mathrm{~min}$ after deferoxamine injection as shown in Fig.1.

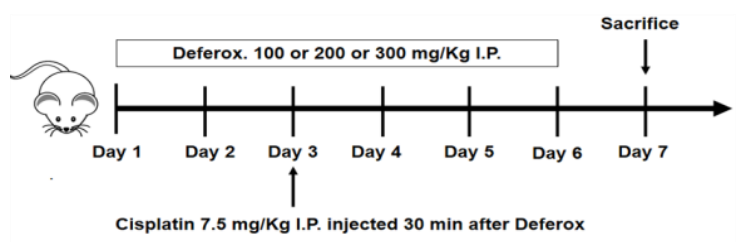

Fig.1. A schematic diagram illustrating the treatment regimen used in rats receiving both deferoxamine and cisplatin

Rats in groups 3, 4 and 5 received deferoxamine i.p. daily for 6 consecutive days at 3 dose levels as follows: 100, 200 and $300 \mathrm{mg} / \mathrm{Kg}$ and on day 3 cisplatin was injected i.p. as a single dose of $7.5 \mathrm{mg} / \mathrm{Kg}$ approximately $30 \mathrm{~min}$ after 
deferoxamine injection. Finally, the rats were sacrificed on day 7.

The used dosage range of deferoxamine is based on the dose previously used to assess deferoxamine's effect in cisplatin-induced lipid peroxidation [10]. On the morning of the seventh day, animals were lightly anesthetized and opened and blood samples were collected from the caudal vena cava to assess the levels of serum creatinine and blood urea nitrogen (BUN). The rats were then euthanized by decapitation and their kidney specimens were collected for histopathological examination.

\subsection{Assessment of nephrotoxicity markers}

Serum creatinine and BUN were assessed using the kits available commercially in the Egyptian market (Biodiagnostic Co., Egypt). The manufacturer's protocol provided with the kits was generally followed.

\subsection{Assessment of kidney index}

Both body weight and kidneys` weight were taken down on the sacrifice day. The kidney index was determined based on the following formula: (kidney weight/total body weight) $\mathrm{x}$ 100.

\subsection{Histopathological examination}

For routine histopathological examination, $10 \%$ formalin was used for fixing the autopsy kidney for $24 \mathrm{~h}$. Tap water was used for washing the kidney specimens then serial dilutions of the following stated alcohols (methyl, ethyl, and absolute ethyl) were utilized for kidney tissue dehydration. Xylene was used for clearing the tissue specimens, then the specimens were embedded for $24 \mathrm{~h}$ in paraffin at $56{ }^{\circ} \mathrm{C}$ placed in a hot air oven. The obtained paraffin blocks were further sectioned at 4 microns using a sledge microtome. Glass slides were used for collecting the deparaffinized tissue sections, followed by staining with hematoxylin and eosin stain for routine light microscopy examination [11].

\subsection{Statistical Analyses}

All statistical analyses were performed using version 3.05 of GraphPad InStat software (GraphPad Software, La Jolla, CA) was used for performing all analyses. Graphs were sketched using version 5.00 of GraphPad Prism software (GraphPad Software, La Jolla, CA). Data are introduced as mean+standard deviation. Individual treatment groups were compared to each other using paired or unpaired Student's ttest as convenient. Multiple comparisons were performed using one-way analysis of variance (ANOVA) then the Tukey-Kramer test was applied for post hoc analysis. The level of statistical significance was adjusted at $\mathrm{P}<0.05$.

\section{RESULTS}

\subsection{The effect of deferoxamine injection on nephrotoxicity markers}

Creatinine is the waste product of muscle metabolism which is normally cleared by the kidneys. The obtained results of serum creatinine measurements are depicted in Fig. 2. Cisplatin only treated rats increased serum creatinine levels significantly compared to the control group by 5.6 folds $(\mathrm{P}<0.001)$. All deferoxamine treated rats (at all dose levels) failed to show any significant reduction in the serum creatinine levels when compared to cisplatin only treated group $(\mathrm{P}>0.15)$. Blood urea nitrogen is another essential renal function test that rises early after kidney injury. Urea is produced in the liver as a waste product of protein digestion and is cleared through the kidneys. The levels of BUN assessed in the different groups are shown in Fig. 3. Rats receiving cisplatin injection only upregulated BUN levels markedly by 4.07 folds $(\mathrm{P}<0.001)$. Treatment of animals with deferoxamine didn't produce any significant downregulation in BUN levels compared to cisplatin only treated animals $(\mathrm{P}>0.8)$ and hence, BUN measurements confirm the results obtained from serum creatinine 
measurements.

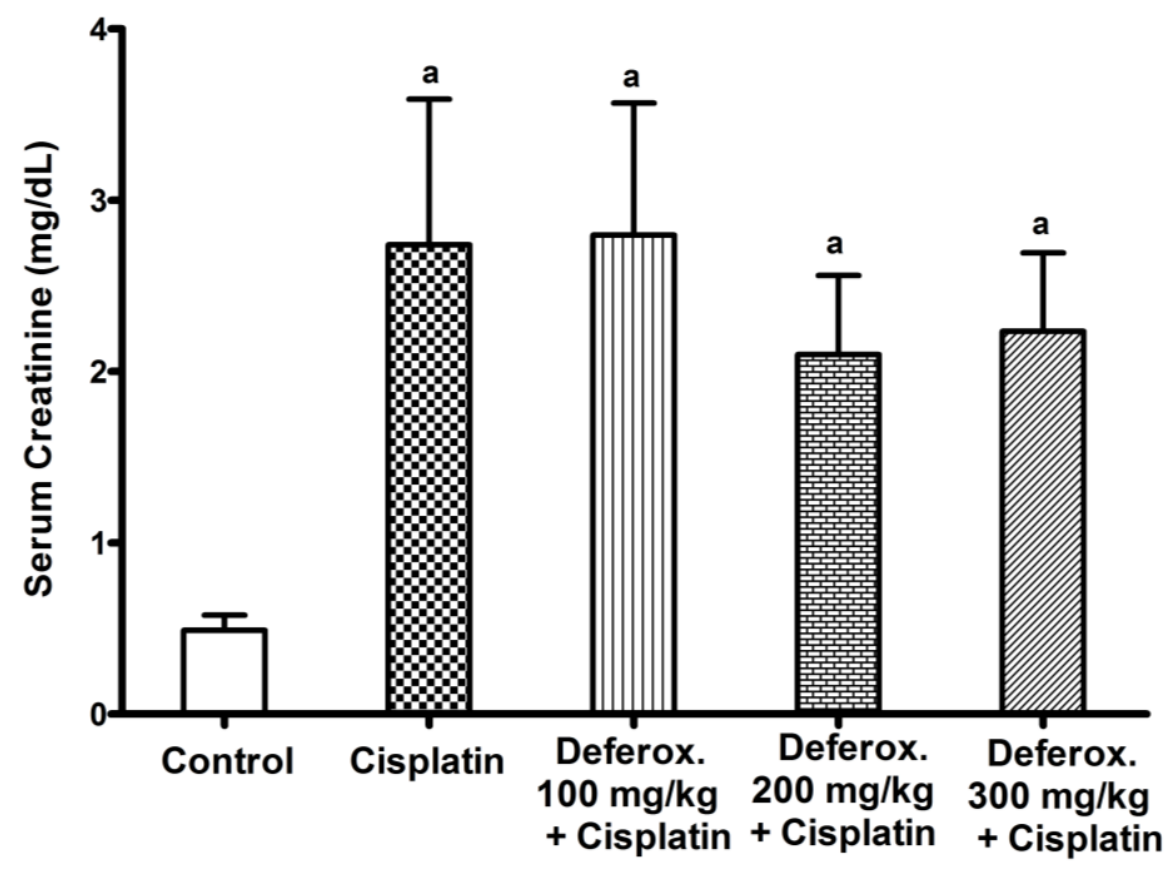

Fig. 2. Effect of cisplatin and pre-treatment of deferoxamine on levels of serum creatinine

Data are represented as means \pm standard deviation (where $n=6-10$ ). a: statistically significant when compared to the control group using ANOVA followed by Tukey-Kramer test as post-hoc test, $\mathrm{P}<0.05$.

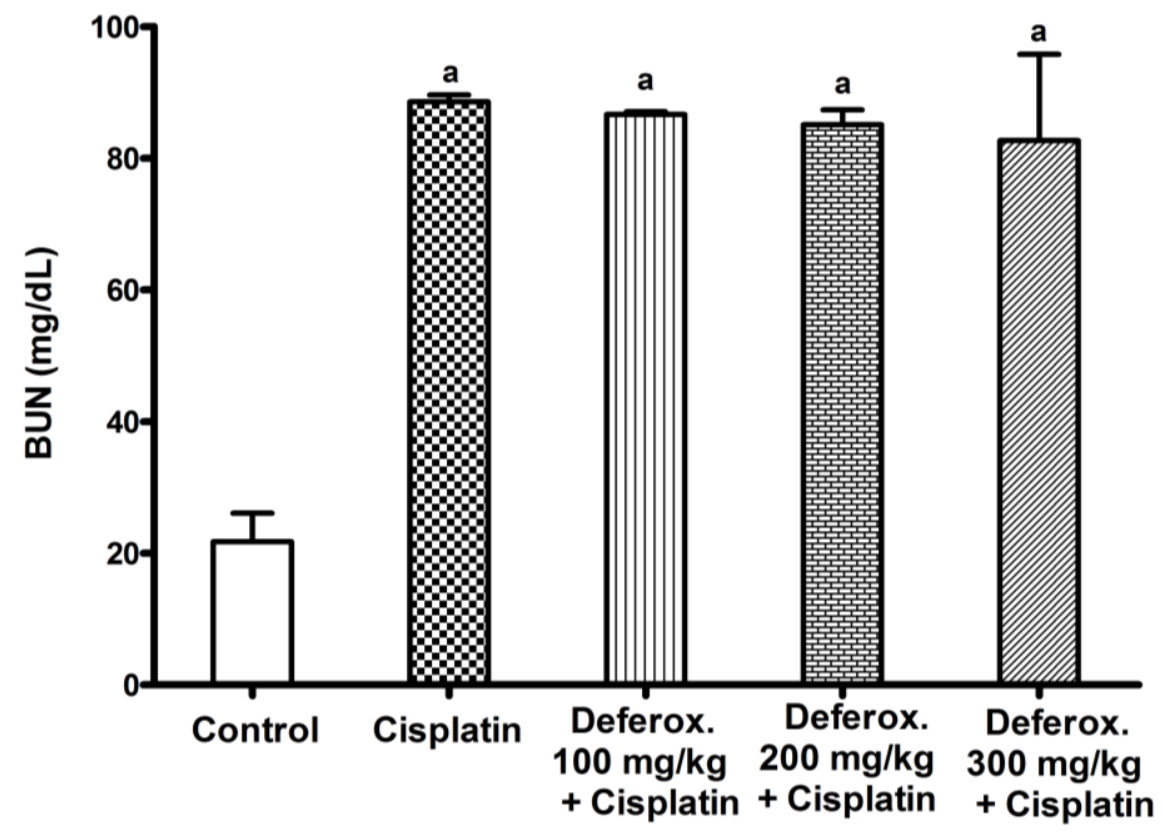

Fig. 3. Effect of cisplatin and pre-treatment of deferoxamine on levels of BUN

Data are represented as means \pm standard deviation (where $n=6-10$ ). a: statistically significant when compared to the control group using ANOVA followed by Tukey-Kramer test as post-hoc test, $\mathrm{P}<0.01$. BUN: Blood Urea Nitrogen. 


\subsection{The effect of deferoxamine injection on mortality rate and kidney index}

Mortality percentages as well as total body weight and kidney indices are shown in Table 1. Mortality rates were high in the cisplatin only treated group $(40 \%)$. Treatment with deferoxamine at the 100 and $200 \mathrm{mg} / \mathrm{Kg}$ dose decreased mortality rate to only $20 \%$, while the high dose of $300 \mathrm{mg} / \mathrm{Kg}$ deferoxamine treatment had a $30 \%$ mortality rate. Treatment with cisplatin, deferoxamine $100 \mathrm{mg} / \mathrm{Kg}$, deferoxamine $200 \mathrm{mg} / \mathrm{Kg}$ and deferoxamine 300 $\mathrm{mg} / \mathrm{Kg}$ increased the kidney indices by 1.49 , $1.51,1.65$, and 1.38 folds respectively. These results are in line with serum creatinine and BUN measurements where all deferoxamine treatments could not produce any significant nephroprotective activity.

Table 1. The results of deferoxamine pre-treatment on mortality rate, body weight changes and kidney index

\begin{tabular}{|c|c|c|c|c|}
\hline \multirow[t]{2}{*}{ Different treatment groups } & \multirow{2}{*}{$\begin{array}{l}\text { Number of } \\
\text { dead rats }\end{array}$} & \multicolumn{2}{|c|}{ Body weight (g) } & \multirow[t]{2}{*}{ Kidney Index } \\
\hline & & Before treatment & After treatment & \\
\hline Control & $0 / 10$ & $137.78 \pm 21.03$ & $161.56 \pm 28.44^{*}$ & $0.37 \pm 0.02$ \\
\hline Cisplatin & $4 / 10$ & $142.64 \pm 16.68$ & $132.91 \pm 15.50^{*}$ & $0.55 \pm 0.07^{\mathrm{a}}$ \\
\hline Cisplatin/ Deferoxamine (100 mg/Kg) & $2 / 10$ & $151.3 \pm 11.05$ & $142.9 \pm 12.36^{*}$ & $0.56 \pm 0.05^{\mathrm{a}}$ \\
\hline Cisplatin/ Deferoxamine $(200 \mathrm{mg} / \mathrm{Kg})$ & $2 / 10$ & $135.75 \pm 8.58$ & $120.5 \pm 9.26^{*}$ & $0.61 \pm 0.04^{\mathrm{a}}$ \\
\hline Cisplatin/ Deferoxamine (300 mg/Kg) & $3 / 10$ & $134.57 \pm 19.79$ & $132.29 \pm 22.82$ & $0.5 \pm 0.07^{\mathrm{a}}$ \\
\hline
\end{tabular}

Data are represented as mean \pm standard deviation (where $n=6-10$ ). a: statistically significant when compared to the control group applying ANOVA followed by Tukey-Kramer test as post-hoc analysis test, $\mathrm{P}<0.05$. *: statistically significant from the values obtained before treatment using paired Student`s t-test, $\mathrm{P}<0.05$.

\subsection{Assessment of kidney histology}

Alternations in kidney histology between the different animal groups are illustrated in Fig. 4 and Table 2. The control group receiving saline showed an intact histological structure in both the glomeruli and the renal tubules with no histopathological alternations (Fig. 4A). On the other side, cisplatin only treated rats showed degeneration and necrosis in the epithelium lining the tubules at the corticomedullary junction along with focal inflammatory cell infiltration as well as the appearance of some eosinophilic casts in the renal tubules of the corticomedullary portion (Fig. 4B). Treatment with deferoxamine $100 \mathrm{mg} / \mathrm{Kg}$ showed tubular degeneration in the corticomedullary region as well as inflammatory cell infiltration (Fig. 4C) and deferoxamine at $200 \mathrm{mg} / \mathrm{Kg}$ showed both tubular degeneration along tubular necrosis (Fig. 4D). By the same token, deferoxamine at 300 $\mathrm{mg} / \mathrm{Kg}$ dose showed an intact histological structure in the cortical region while both tubular degenerations, as well as eosinophilic casts, were prominent in the corticomedullary junction (Fig. 4E). 


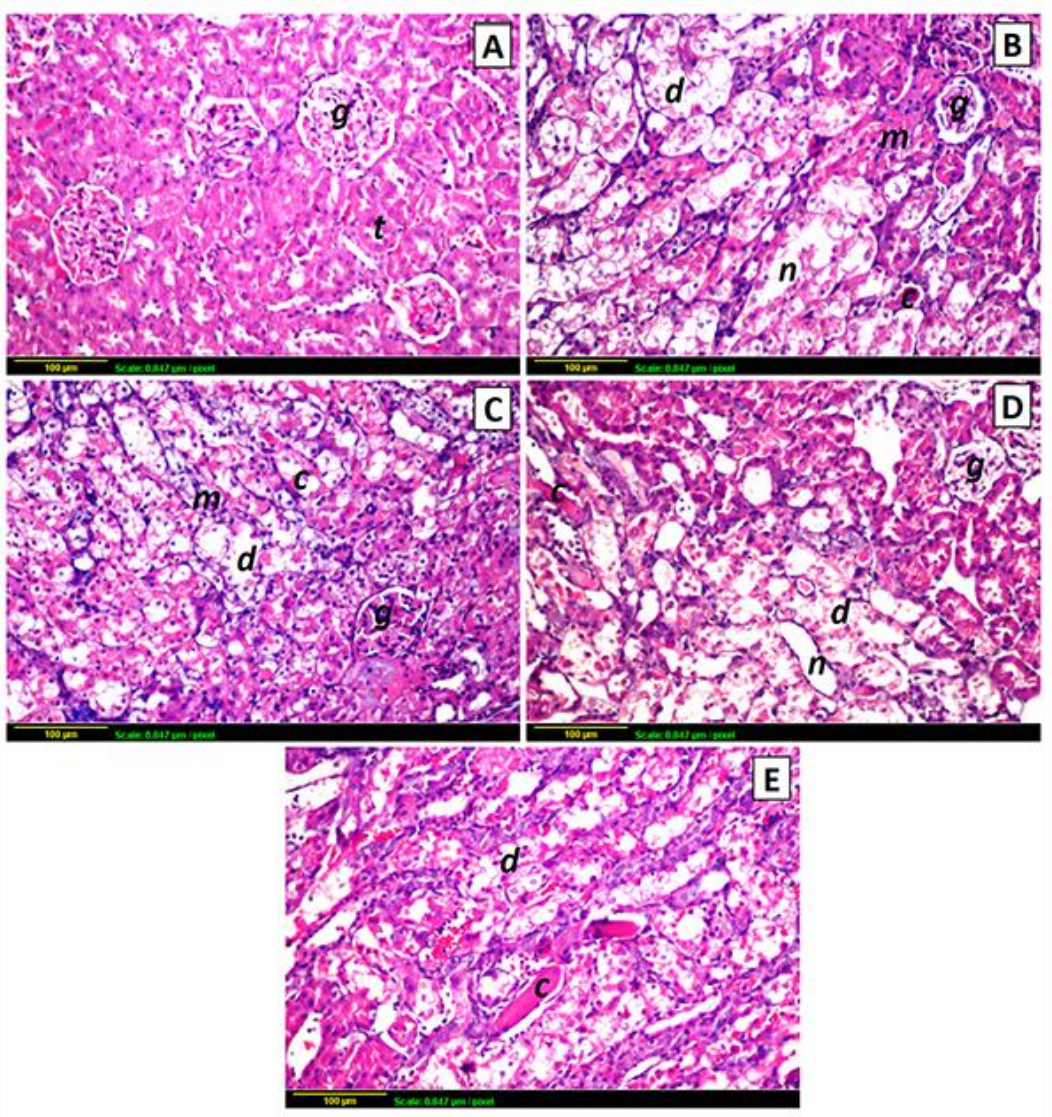

Fig. 4. Representative photomicrographs of kidney sections stained by hematoxylin and eosin stain

A: Control group representing an intact glomeruli $(\mathrm{g})$ and normal histological architecture of the tubules ( $\mathrm{t}$ ) at the cortical region (x 400). B: Cisplatin only treated group showing tubular degeneration (d) and necrosis (n) along with eosinophillic casts (c) and inflammatory cell infilteration $(\mathrm{m})$ in the corticomedullary junction (x 400). C: Deferoxamine pre-treated group (100 mg/Kg) showing tubular degeneration (d), eosinophillic casts (c) along with inflammatory cell infilteration (m) in the corticomedullary junction (x 400). D: Deferoxamine pre-treated group (200 mg/Kg) showing tubular degeneration (d), eosinophillic casts (c) along with tubular necrosis $(\mathrm{n})$ in the corticomedullary junction (x 400). E: Deferoxamine pre-treated group (300 mg/Kg) showing tubular degeneration (d) along with eosinophillic casts (c) within medullary tubules (x 400).

Table 2. Histopathological variations occurring in kidney samples obtained from the different treated animal groups

\begin{tabular}{lccccc}
\hline $\begin{array}{l}\text { Histopathological } \\
\text { variations }\end{array}$ & Control & Cisplatin & $\begin{array}{c}\text { Cisplatin/ } \\
\text { Deferoxamine } \\
(\mathbf{1 0 0} \mathbf{~ m g} / \mathbf{K g})\end{array}$ & $\begin{array}{c}\text { Cisplatin/ } \\
\text { Deferoxamine } \\
(\mathbf{2 0 0} \mathbf{~ m g} / \mathbf{K g})\end{array}$ & $\begin{array}{c}\text { Cisplatin/ } \\
\text { Deferoxamine } \\
(\mathbf{3 0 0} \mathbf{~ m g} / \mathbf{K g})\end{array}$ \\
\hline $\begin{array}{l}\text { Degeneration of } \\
\text { renal tubules }\end{array}$ & - & +++ & +++ & +++ & ++ \\
$\begin{array}{l}\text { Necrosis of renal } \\
\text { tubules }\end{array}$ & - & +++ & ++ & +++ & ++ \\
$\begin{array}{l}\text { Presence of renal } \\
\text { casts }\end{array}$ & - & ++ & ++ & ++ & +++ \\
$\begin{array}{l}\text { Infilteration by } \\
\text { inflammatory cells }\end{array}$ & - & ++ & ++ & + & + \\
\hline
\end{tabular}

+++ Severe. ++ Moderate. + Mild. -None. 


\section{DISCUSSION}

Cisplatin is one of the major used antineoplastic agents. Its efficacy along with its relatively low price makes it one of the most important chemotherapeutic agents available in Egypt and most developing countries. However, cisplatin-induced acute renal injury remains a major dose-limiting side effect. Numerous research articles have discussed the molecular mechanisms underlying cisplatin-induced acute kidney failure as well as the potential nephroprotective strategies $[\mathbf{3}, \mathbf{1 2}, \mathbf{1 3}]$.

One key study provided both in vitro and in vivo evidence suggesting that the exposure of the kidney to cisplatin increases the labile iron content within the renal tubules which contributes to oxidative kidney damage and suggesting a possible nephroprotective effect of iron chelators [9]. Deferoxamine is one of the classical iron chelators readily available in the Egyptian market. Hence, the current research study aimed to question the potential nephroprotective effect of a single daily intraperitoneal dose of deferoxamine at three different dose levels in an acute model of cisplatin-induced nephrotoxicity.

Cisplatin only treated mice had the highest mortality percentages. Treatment with deferoxamine decreased mortality in mice by around $50 \%$. Measurement of kidney indices and serum creatinine and BUN levels showed the absence of deferoxamine nephroprotective activity at the used doses and animal model. The histopathological examination further confirmed serum creatinine and BUN measurements; where most animal specimens showed tubular degeneration and the presence of eosinophilic casts highlighting the severe damage occurring in the kidney tubules due to cisplatin administration and lack of significant deferoxamine nephroprotection.
Previously, a daily intraperitoneal injection of deferoxamine has shown a nephroprotective effect in a sub-acute model of cisplatin nephrotoxicity [14]. Moreover, administration of deferoxamine by osmotic pump produced a significant nephroprotection in acute cisplatin nephrotoxicity [9]. In accordance with our results, a research study showed the lack of deferoxamine nephroprotection in a comparable model to the one used in our study, however, this study showed that deferoxamine was capable of reducing lipid peroxidation within the kidneys of treated animals [15]. The present study has a major significance where it shows that the iron chelation capacity of deferoxamine provided by single daily dosing is insufficient to preserve kidney function in severe animal models of acute cisplatin nephrotoxicity, this besides the relatively high dose $(300 \mathrm{mg} / \mathrm{kg})$ used for administrating deferoxamine in the present research work provides the basis for the inapplicability of using deferoxamine as an adjunct therapy to cisplatin chemotherapeutic regimens. The strong evidence available on the role of catalytic iron in kidney diseases as well as cisplatin nephrotoxicity along with the inconsistent results obtained from the administration of deferoxamine puts the chelating capacity of deferoxamine under question especially when it is administered as a single dose daily. Deferoxamine has a very short halflife [16] and it could be possible that the chelator is quickly cleared from plasma before achieving the desired iron-chelating function. This might also explain the success of models administrating deferoxamine through an osmotic pump where a constant infusion and sustainable plasma concentrations are maintained.

\section{Conclusion and Recommendations}

In conclusion, the current study shows that giving deferoxamine as a single daily intraperitoneal injection is not protective in 
cisplatin-induced acute renal failure. Iron homeostasis has developed as a potential therapeutic target in acute renal injury [17]. Hence, the authors suggest the study and investigation of the potential nephroprotective effect of alternative iron chelators that could have more favorable pharmacokinetics and iron chelation capacities when administered daily in reasonable doses such as the oral iron chelator 'deferiprone'.

\section{Declarations}

\section{Ethics Approval and Consent to Participate}

The research protocol and all procedures of experimentation have been approved by the research ethics committee of The School of Pharmacy, Ain Shams University. The ethics committee number approving the protocol of the present study is 29. During the experimentation period, all efforts were put together to keep animal suffering the minimal as possible.

\section{Consent to Publish}

The final manuscript was approved by all authors for publishing in the APS journal.

\section{Availability of Data and Materials}

All generated or analyzed data during the present research study are included in the main manuscript of this original research article.

\section{Competing Interests}

There are no competing interests to be declared.

\section{Funding statement}

No funding was received by any of the authors for conducting the experiments.

\section{Authors' contributions}

M.A. purchased chemicals and animals, designed the experiments, performed the experiments, gathered and analyzed the results, and put down together the first draft of the research manuscript; E.M. developed the research conception and idea, provided the main experimental design, shared in providing materials and chemicals, supervised and shared in the practical experimentation and data analyses and revised and approved the final version of the manuscript. R.E., S.A, and E.E. guided and followed the practical experiments and data analyses and revised and approved the final version of the research manuscript; M.E. guided and followed the practical experimentation and revised and approved the final version of the research manuscript and followed it up for publication.

\section{Acknowledgment}

The authors highly acknowledge the support and help of all colleagues at the Department of Pharmacology and Toxicology, School of Pharmacy, Ain Shams University.

\section{REFERENCES}

1. Holditch SJ, Brown CN, Lombardi AM, Nguyen KN, Edelstein CL. Recent Advances in Models, Mechanisms, Biomarkers, and Interventions in Cisplatin-Induced Acute Kidney Injury. Int J Mol Sci 2019; 20:3011. DOI: $10.3390 / \mathrm{ijms} 20123011$

2. Santos N, Ferreira RS, Santos ACD. Overview of cisplatin-induced neurotoxicity and ototoxicity, and the protective agents. Food Chem Toxicol 2020;136:111079. DOI: 10.1016/j.fct.2019.111079

3. Duffy EA, Fitzgerald W, Boyle K, Rohatgi R. Nephrotoxicity: Evidence in Patients Receiving Cisplatin Therapy. Clin J Oncol Nurs 2018; 22:175-83. DOI: 10.1188/18.CJON.175-183

4. Helfrich KK, Saini N, Kling PJ, Smith SM. Maternal iron nutriture as a critical modulator of fetal alcohol spectrum disorder risk in alcohol-exposed pregnancies. Biochem Cell Biol 2018; 96:204-12. DOI: 10.1139/bcb- 
2017-0206

5. Yiannikourides A, Latunde-Dada GO. A Short Review of Iron Metabolism and Pathophysiology of Iron Disorders. Medicines (Basel) 2019; 6:85. DOI: 10.3390/medicines6030085

6. Mezzaroba L, Alfieri DF, Colado Simao AN, Vissoci Reiche EM. The role of zinc, copper, manganese, and iron in neurodegenerative diseases. Neurotoxicology 2019; 74:230-41. DOI: 10.1016/j.neuro.2019.07.007

7. Ali MK, Kim RY, Brown AC, Donovan C, Vanka KS, Mayall JR, et al. Critical role for iron accumulation in the pathogenesis of fibrotic lung disease. J Pathol 2020; 251:4962. DOI: $10.1002 /$ path. 5401

8. Swaminathan S. Iron Homeostasis Pathways as Therapeutic Targets in Acute Kidney Injury. Nephron 2018; 140:156-9. DOI: $10.1159 / 000490808$

9. Baliga R, Zhang Z, Baliga M, Ueda N, Shah $\mathrm{SV}$. In vitro and in vivo evidence suggesting a role for iron in cisplatin-induced nephrotoxicity. Kidney Int 1998; 53:394-401. DOI: 10.1046/j.1523-1755.1998.00767.x

10. Kadikoylu G, Bolaman Z, Demir S, Balkaya M, Akalin N, Enli Y. The effects of desferrioxamine on cisplatin-induced lipid peroxidation and the activities of antioxidant enzymes in rat kidneys. Hum Exp Toxicol 2004; 23:29-34. DOI: 10.1191/0960327104ht413oa

11. Banchroft JD, Stevens A, Turner DR. Theory and practice of histological techniques. 4th ed. New York, London, San Francisco, Tokyo: Churchil Livingstone Elsevier; 1996.

12. Crona DJ, Faso A, Nishijima TF, McGraw KA, Galsky MD, Milowsky MI. A Systematic Review of Strategies to Prevent CisplatinInduced Nephrotoxicity. Oncologist 2017;
22:609-19. DOI: 10.1634/theoncologist.20160319

13. Zhu L, Yuan Y, Yuan L, Li L, Liu F, Liu J, et al. Activation of TFEB-mediated autophagy by trehalose attenuates mitochondrial dysfunction in cisplatin-induced acute kidney injury. Theranostics 2020; 10:5829-44. DOI: 10.7150/thno.44051

14. Ozdemir E, Dokucu AI, Uzunlar AK, Ece A, Yaldiz M, Ozturk H. Experimental study on effects of deferoxamine mesilate in ameliorating cisplatin-induced nephrotoxicity. Int Urol Nephrol 2002; 33:127-31. DOI: 10.1023/a:1014442027991

15. al-Harbi MM, Osman AM, al-Gharably NM, al-Bekairi AM, al-Shabanah OA, Sabah DM, et al. Effect of desferrioxamine on cisplatininduced nephrotoxicity in normal rats. Chemotherapy 1995; 41:448-54. DOI: $10.1159 / 000239381$

16. Cunningham MJ, Nathan DG. New developments in iron chelators. Curr Opin Hematol 2005;12:129-34. DOI: 10.1097/01.moh.0000152631.63469.07

17. Walker VJ, Agarwal A. Targeting Iron Homeostasis in Acute Kidney Injury. Semin Nephrol 2016; 36:62-70. DOI: 10.1016/j.semnephrol.2016.01.003 